\title{
A GRAVIDEZ ECTÓPICA E O CONTRACEPTIVO ORAL EMERGENCIAL
}

\author{
Tathiana Costacoi ${ }^{1}$
}

RESUMO: A gravidez ectópica (GE) ocorre em local extrauterino, por diversas causas, caracterizando os maiores índices de mortalidade materna no primeiro trimestre gestacional e em muitos casos, necessita de tratamento cirúrgico e/ou emergencial. O presente estudo reflete acerca de sua ocorrência após o uso do contraceptivo oral de emergência. Evidenciou-se a importância da adesão ao planejamento familiar, diagnóstico precoce, maior conhecimento por parte das usuárias e parceiros, além de atenção por parte dos profissionais da saúde para promoção de orientações e assistência mais eficazes.

Descritores/ Palavras-Chave: Gestação de alto risco; Gravidez tubária; Gravidez ectópica; Gestação Ectópica, Contracepção feminina.

ABSTRACT: Ectopic pregnancy (EG) occurs in an extrauterine location for several causes, characterizing the highest rates of maternal mortality in the first gestational trimester and in many cases, requires surgical and / or emergency treatment. The present study reflects on its occurrence after emergency oral contraceptive use. The importance of adherence to family planning, early diagnosis, greater knowledge by users and partners, and attention by health professionals to promote more effective guidance and assistance were highlighted.

Keywords/ Descriptions: High risk pregnancy; Tubal pregnancy; Ectopic pregnancy; Ectopic Gestation, Female Contraception.

\section{INTRODUÇÃO}

A Organização Mundial da Saúde (OMS) é responsável pela contribuição em ações que visem a redução da mortalidade materna. Embora entre os anos de 1990 e 2015 as taxas tenham sido reduzidas em $44 \%$, diariamente ainda ocorrem aproximadamente 830 óbitos

\footnotetext{
${ }^{1}$ Graduada em Enfermagem, especializada em Docência em Enfermagem pela Universidade Anhembi Morumbi e pós graduanda em Enfermagem Obstétrica. Instituição: UNESA ( Universidade Estácio de Sá, pós graduação em Enfermagem Obstétrica)-E- mail: tathicostacoi@gmail.com.
} 
maternos por causas evitáveis, relacionados ao processo da gestação ou parto; sendo $99 \%$ deles nos países em desenvolvimento. (OPAS, 2019). Dentre as causas evitáveis encontramos as gestações ectópicas, ocorridas em $95 \%$ dos casos na tuba uterina e que são a causa de mortalidade materna mais frequente durante os primeiros 3 meses de gestação e representam I em cada roo gestações, com taxa de mortalidade de I,8 óbitos para cada Iooo casos; nos últimos anos o aumento de casos é singularmente notório, juntamente com a investigação de novos fatores de risco, como as tecnologias, cada vez mais avançadas para reprodução assistida. (NÓBREGA; 2015)

Uma gravidez ectópica (GE) pode ser definida como extrauterina, ou seja, ocorre quando a implantação do ovo ocorre fora da cavidade uterina e ameaça a vida da mulher, necessitando de rápido diagnóstico. (MONTENEGRO; REZENDE FILHO, 2017). Não há dados específicos relacionados à prevalência de GE em níveis nacional e/ou regionais no Brasil, mas possivelmente são variados uma vez que somos parte de uma sociedade com elevados índices de mulheres em idade reprodutiva, uso de Dispositivo Intrauterino (DIU) e atividade sexual favorável à inúmeras infecções do trato reprodutivo - o que salienta a importância da prevenção, investigação e possíveis tratamentos precoces avistando menores índices de tratamentos invasivos e consequentes da GE. (FERNANDES; MORETTI; OLIVOTTI, 2007)

Certos casos de GE podem estar relacionados com os métodos contraceptivos de emergência (CE), habitualmente favoráveis em episódios de relações sexuais desprotegidas entre o ciclo menstrual, por isso se faz necessária atenção e identificação precoce de sua sintomatologia para melhores abordagens terapêuticas. (ZUCCHI et al., 2004) Sendo assim, a relação sexual desprotegida atrelada ao não planejamento de uma gestação e desconhecimento da mulher, podem resultar na escolha do uso de contraceptivos emergenciais, que por sua vez podem resultar no diagnóstico de uma gestação ectópica.

A anticoncepção oral de emergência é de direito da mulher e tem por finalidade a 
precaução de uma gestação indesejada, impedindo que a fecundação ocorra, porém, para o uso correto se faz necessário que o profissional de saúde instrua e auxilie esta mulher, por muitas vezes desinformada quanto aos métodos corretos contraceptivos. (GARCIA; OLIVEIRA; RESENDE, 2015). O cuidado à saúde da mulher necessita de atenção contínua também no que diz respeito a informações sobre os adequados métodos de contracepção, saúde sexual e autonomia sobre seu próprio corpo.

Diante deste panorama emerge uma questão norteadora: Qual a produção científica na área da saúde acerca da gestação ectópica como consequência pelo uso do contraceptivo oral de emergência? Para a realização desta pesquisa traçou-se como objetivo: Refletir acerca da gestação ectópica como consequência pelo uso do contraceptivo oral de emergência, a partir de produções científicas. Entende-se que este fato pode acarretar procedimentos cirúrgicos emergenciais como a laparotomia, videolaparoscopia, salpingostomia e salpingectomia; bem como hemorragias e consequente aumento da mortalidade materna (MONTENEGRO; REZENDE FILHO, 2017). A motivação do estudo surge de uma realidade vivenciada pela autora e sua relevância se dá devido à escassez de estudos científicos sobre a temática no

Brasil, salientando a importância da atenção na saúde da mulher e consequente redução da 846 mortalidade materna. Tal temática vai de encontro a Agenda Nacional de Prioridades de Pesquisa em Saúde do Ministério da Saúde, que apresenta um rol de subagendas, e dentre elas o tópico sobre a "Mortalidade materna e fatores de vulnerabilidade" (BRASIL, 20I8).

\section{METODOLOGIA}

Estudo bibliográfico baseado em referências já publicadas anteriormente; de natureza qualitativa, que no campo da saúde, procura compreender significados do processo saúde-doença a fim de melhorar o conjunto profissional-paciente-família-instituição (TURATO; 2005), fundamentado na reflexão a partir de publicações acerca da temática disponibilizadas na Biblioteca Virtual de Saúde (BVS). 


\section{O contraceptivo oral de emergência e a gestação ectópica.}

No ano de 2013, estima-se que houve mundialmente 289 mil óbitos maternos, o que representa uma vida a cada 2 minutos, apenas relacionados à gravidez e parto; além das notórias 225 milhões de mulheres que não obtiveram acesso a um planejamento familiar. Dentre as novas estratégias mundiais da saúde iniciadas em 2016 e com visão até 2030, está a redução da mortalidade materna e dentre as principais intervenções relacionadas à saúde da mulher estão o acompanhamento e informações sobre saúde sexual e reprodutiva, incluindo métodos de contracepção. (ONU, 2015)

A incidência de casos de gestação ectópica após a contracepção emergencial (CE) não pode ser contabilizada, uma vez que a população usuária do método não é estreitamente conhecida, entretanto é necessário que as usuárias tenham acesso à informação de que este não é um método totalmente seguro e que possui riscos incluindo a gestação tubária. (ROSA; ANRIQUE; RODRÍGUEZ, 2009). Este fato sinaliza a importância em contabilizar a população usuária do método e a realidade dos possíveis efeitos que causa.

O componente comumente encontrado nas pílulas do dia seguinte é o Levonorgestre ${ }^{\circledR}$ (LNG), que aumenta o risco de gestação na tuba uterina quando falha como contraceptivo emergencial (VALENZUELA, 2005), além de modificar a motilidade tubária, tornando difícil a passagem do espermatozoide e óvulo. (LIBBS, 2019).

O LNG é um anticoncepcional hormonal que atua até 120 horas após a relação sexual desprotegida, através da suspensão da ovulação e migração do esperma, tendo indicação reservada a situações especiais e de exceção, como a relação sexual inesperada sem uso de método anticoncepcional, falha ou uso inadequado do método, ou em casos de violência sexual. (PAIVA; BRANDÃO, 2017)

Embora seja caracterizada como direito sexual e reprodutivo feminino, a CE com LNG inclusa na lista de medicamentos essenciais da OMS desde o ano de 1999, repercute em oposições uma vez em que é indicada visando a impedição de um aborto futuro, ocasionando a morte de um ser real que, para a imposição já é hipoteticamente um ser viável. 
(PRETELL-ZARATE; 2013). Sendo assim, as investigações a respeito de seus efeitos tampouco deixarão de ser questionadas pois compreendem o encontro de gametas femininos e masculinos que carregam consigo material hereditário, que constitui múltiplos sentidos para cada ser humano. (COMISIÓN DE ÉTICA DE LA FACULTAD DE MEDICINA DE LA UNIVERSIDAD DE CHILE, 2005)

Atualmente no Brasil, a CE está disponível desde a atenção básica até a sua venda livre no comércio farmacêutico, porém, ainda é vinculada aos sentimentos de vergonha e imoralidade no ato de sua compra, historicamente retornando ao sexo frágil feminino, uma vez em que a dificuldade inicia-se em exercer sua autonomia até no que diz respeito aos direitos sexuais e reprodutivos. (PAIVA; BRANDÃO, 2017)

No tocante aos Direitos Sexuais e reprodutivos, a população feminina do país apresenta dificuldades em planejar a reprodução, juntamente com seu parceiro e por este motivo, o planejamento familiar é imprescindível. Através do planejamento, é possível compreender que a satisfação de seus desejos sexuais se difere do anseio pela maternidade, permitindo ao casal a liberdade de escolha em ter filhos ou não, bem como o período certo 848 para que este momento aconteça. (GARCIA; OLIVEIRA; RESENDE, 2015)

Para as que seriam relevantes à clínica do desejo, sabe-se que cada vez menos mulheres reconhecem na condição de mãe a finalidade de suas vidas, que em vários países ocorre o adiamento da primeira maternidade e que continua a diminuir o número médio de filhos por mulher. (...) Essas tendências sugerem que ter filho é uma opção e que está potencialmente em conflito com os sonhos profissionais e com o desejo de aproveitar a vida enquanto der (MELO; VAZ, 2019).

A fim de proporcionar maiores informações a mulher, são imprescindíveis maiores conhecimentos por parte dos profissionais de saúde e até da população, dados a respeito da gestação ectópica e suas causalidades, para melhorias em sua prevenção e detecção precoce, mesmo que a mulher não tenha iniciado o seu pré-natal, resultando em uma assistência conhecedora e habilidosa, além da diminuição dos casos que resultam em graves complicações. (CAMPOS et al., 2012) 


\section{As possíveis consequências e complicações para a população feminina.}

Reconhecer que a contracepção emergencial pode acarretar efeitos adversos inesperados e até irreparáveis como a GE na tuba uterina, muitas vezes desconhecidos pelas usuárias e favorece maior atenção por parte das mesmas no momento de escolha quanto ao utilizá-lo ou não.

Quanto a GE tubária, nos casos de não diagnóstico e/ou tratamento precoces, podem ocorrer a ruptura da tuba e/ou sangramento na cavidade abdominal, implicando em intervenções como a videolaparoscopia ou cirurgia aberta; porém em casos em que não há evidências de sangramento e os níveis hormonais estão relativamente baixos, o uso do Metotrexato ${ }^{\circledR}$ em via intramuscular pode ser considerado, além de conduta expectante. (HAJENIUS et al., 2007)

O desfecho de uma GE sempre dependerá de sua localização, diagnóstico de suas formas clínicas como a subaguda, aguda ou abdominal, além da sintomatologia, estado hemodinâmico da paciente e níveis do hormônio BetaHCG, necessitando de criteriosa 849 avaliação clínica e diagnóstico por imagem confirmado para decisão de conduta (MONTENEGRO; REZENDE FILHO, 2017).

As consequências de uma GE podem ir além de modificações físicas ou hormonais, podendo afetar o seu lado psicológico e gerando a vivência de um luto, mesmo por um filho não idealizado. Segundo MELO; VAZ, 2019, "Sem o reconhecimento da existência desses seres de vida breve, consequentemente, não se reconhece a dor da perda, sendo essa a principal queixa daqueles cujos bebês morreram prematuramente”.

Uma gestação não planejada pode ser vivenciada pela mulher com certa opressão, gerando conflitos entre a cobrança da maternidade culturalmente imposta em nossa sociedade e a frustração em não desejar vivenciá-la, atrelado ao fato de que no Brasil a prática do aborto 
é crime e só pode ser realizada em casos específicos e previstos em lei. (ROCHA et al., 2013)

Durante o processo da perda gestacional, desejada ou não, torna a mulher vulnerável e reflete-se em intensas reações emocionais que caracterizam grande risco de depressão, estresse e culpa, podendo ainda aumentar o risco ao suicídio (BENUTE et al., 2016). A intitulação de mãe para paciente, quase que imposta de maneira derrisória conseguinte da própria maternidade é atrelada à conflitos e culpas, em busca de porquês, muitas vezes suavizados com a controvérsia d recuperação de sua própria vida. (ROCHA et al., 2013)

\section{CONSIDERAÇÕES FINAIS}

A GE necessita de cautela e diagnóstico precoce devido aos riscos que carrega para a vida da mulher, independente a uma gestação planejada ou não. Houve grande dificuldade em encontrar referências que a correlacionasse com o uso do contraceptivo oral de emergência, realidade ainda pouco evidenciada no Brasil, onde é distribuído e comercializado até mesmo sem prescrição ou orientação por parte dos profissionais de saúde a respeito de suas possíveis consequências, sendo a gestação tubária uma delas.

Através das reflexões acerca da temática, o estudo evidenciou que ainda se faz 850 necessária adesão ao planejamento familiar, maior conhecimento por parte das usuárias do método juntamente com seus parceiros, de modo em que o utilizem de maneira não habitual e atenção por parte dos profissionais de saúde, que devem orientar a saúde sexual e reprodutiva da mulher, bem como dar atenção ao lado psicológico e emocional que pode ser afetado neste momento.

\section{REFERÊNCIAS}

BENUTE, Gláucia Rosana Guerra et al . Depression, stress and guilt are linked to the risk of suicide associated to ectopic pregnancy. MedicalExpress (São Paulo, online), São Paulo, v. 3, n. 3, Mi60307, June $2016 \quad$ Available from <http://www.scielo.br/scielo.php?script=sci_arttext\&pid=S2358-04292016000300007\&lng=en\& 
nrm=iso >. access on 13 Dec. 2020. http://dx.doi.org/ro.5935/MedicalExpress.2016.03.07.

BRASIL. MINISTÉRIO DA SAðDE. (Org.). Agenda de Prioridades de Pesquisa do Ministério da Saúde.2018. Disponível em: $\langle$ http://bvsms.saude.gov.br/bvs/publicacoes/agenda_prioridades_pesquisa_ms.pdf $\rangle$. Acesso em: I4 dez. 2020.

CAMPOS, Layna de Cássia Oliveira et al. Perfil socioepidemiológico de mulheres acometidas por gravidez ectópica atendidas em um hospital público de referência em gestação de alto risco na cidade de Belém, Estado do Pará, Brasil. Revista Pan-amazônica de Saúde, [s.1.], v. 3, n. 4 , p.35-42, dez. 2012. Instituto Evandro http://dx.doi.org/10.5123/s2176-62232012000400004.

COMISIÓN DE ÉTICA DE LA FACULTAD DE MEDICINA DE LA UNIVERSIDAD DE CHILE (Chile) (Org.). Levonorgestrel aspectos bioéticos implicadosen su indicación y proscripción. Revista Médica de Chile, [s.1.], v. 133, n. 7, p.84I-846, jul. 2005. SciELO Comision Nacional de Investigacion Cientifica Y Tecnologica (CONICYT). http://dx.doi.org/ı0.4067/s0034-98872005000700013.

FERNANDES, Arlete Maria dos Santos; MORETTI, Tomás Bernardo Costa; OLIVOTTI, Bruna Romano. Aspectos epidemiológicos e clínicos das gestações ectópicas em serviço universitário no período de 2000 a 2004. Revista da Associação Médica Brasileira, [s.1.], v. 53, n. 3, p.213-216, jun. 2007. Elsevier BV. http://dx.doi.org/ro.159o/so104-42302007000300017.

GARCIA, Francielli GonÇalves; OLIVEIRA, Ingrady; RESENDE, Deise. CONHECIMENTO DE MULHERES EM IDADE FÉRTIL SOBRE O USO DA PÍLULA DO DIA SEGUINTE COMO MÉTODO CONTRACEPTIVO EMERGENCIAL. Revista CientÍfica do Instituto Ideia, Rio de Janeiro, v. 2, n. I, p.99-III, mar. 2015. Disponível em: 〈http://www.ideiaeduc.com.br/uploads/revista/pdf/desm/6N.02.2015/6N.02(2015)_Ir.Sa\%C 3\%BAde.DesafiosEnfermeiro.pdf $>$. Acesso em: is dez. 2020. 
HAJENIUS, Petra J et al. Interventions for tubal ectopic pregnancy. Cochrane Database Of $\begin{array}{lllll}\text { Systematic } & \text { Reviews, } & \text { [s.1.], } & \text { jan. } & \text { 2007. Wiley. }\end{array}$ http://dx.doi.org/10.1002/14651858.cdooo324.pub2.

LIBBS (São Paulo) (Org.).POZATO ${ }^{\circledR}$ UNI.Disponível em: <https://www.libbs.com.br/wp-content/uploads/2015/12/Pozato-Uni-versa\%C2\%A6\%C3\%A 20-7_profissional_ampliada.pdf>. Acesso em: I4 dez. 2020.

MELO, Cristina Teixeira Viera de; VAZ, Paulo Roberto Gibaldi. Perda gestacional e neonatal, um sofrimento como outro qualquer. Matrizes, [s.1.], v. 13, n. 2, p.91-II2, 2 set. 2019. Universidade de Sao Paulo Sistema Integrado de Bibliotecas - SIBiUSP. http://dx.doi.org/ıo.II6o6/issn.I982-8I6o.vi3i2p9I-II2.

MONTENEGRO, Carlos Antonio Barbosa; REZENDE FILHO, Jorge de. REZENDE OBSTETRÍCIA FUNDAMENTAL. I4. ed. Rio de Janeiro: Guanabara Koogan, 2017. p. $363-380$

NÓBREGA, Carla. Ameaça de aborto: Gravidez Ectópica. 2015. Disponível em: 852 〈https://www.invitra.pt/gravidez-ectopica/>. Acesso em: 25 abr. 2020.

Organização das Nações Unidas (Org.). ESTRATEGIA MUNDIAL PARA LA SALUD DE LA MUJER, EL NIÑO Y EL ADOLESCENTE (2016-2030): SOBREVIVER, PROSPERAR E TRANSFORMAR. 2015. Disponível em: <https://www.who.int/maternal_child_adolescent/documents/estrategia-mundial-mujer-nin o-adolescente-2016-2030.pdf?ua=I $>$. Acesso em: o8 abr. 2020.

PAIVA, Sabrina Pereira; BRANDÃO, Elaine Reis. Silêncio e vergonha: contracepção de emergência em drogaria do Rio de Janeiro. Revista Estudos Feministas, [s.1.], v. 25, n. 2, p.617-636, 2017. ago. FapUNIFESP (SciELO). http://dx.doi.org/10.159o/r8o6-9584.2017v25n2p617. 
PRETEll-ZARATE, Eduardo A.. Política de anticoncepción oral de emergencia: La experiencia peruana. Rev. perú. med. exp. salud publica, Lima , v. 30, n. 3,p. 487-493, jul. 2013 . Disponible en 〈http://www.scielo.org.pe/scielo.php?script=sci_arttext $\&$ pid $=S_{1726-46342013000300019} \& \operatorname{lng}=e$ s\&nrm=iso >. accedido en II dic. 2020

Recommendations from the German Federal Chamber of Pharmacists [Bundesapothekerkammer].. Pharmacy Practice, [s.1.], v. I4, n. 3, p.I-6, 30 set. 2016. Centro de $\begin{array}{lllll}\text { Investigaciones } & \mathrm{y} & \text { Publicaciones } & \text { Farmaceuticas }\end{array}$ http://dx.doi.org/10.18549/pharmpract.2016.03.828.

ROSA, Guillermo; ANRIQUE, Denisse; RODRÍGUEZ, Paola. ANTICONCEPCIÓN HORMONAL DE EMERGENCIA Y EMBARAZO ECTÓPICO. CASO CLÍNICO. Revista Chilena Obstetrícia e Ginecologia, Chile, v. I, n. 74, p.39-41, dez. 2009.

ROCHA, Glauco Heirison dos S. et al . Gestação ectópica: compreensão e crenças a respeito do diagnóstico, tratamento e suas repercussões. Psicol. hosp. (São Paulo), São Paulo, v. II, n. 2, p. 02-26, jul. $2013 \quad$ Disponível em 853 $<$ http://pepsic.bvsalud.org/scielo.php?script=sci_arttext\&pid=SI677-74092013000200002\&lng= pt\&nrm=iso>. acessos em 28 abr. 2020

SAÚDE. ORGANIZAÇÃO PANAMERICANA DA SAÚDE. (Org.). Folha InformativaMortalidade Materna. Disponível em: <https://www.paho.org/bra/index.php?option=com_content $\&$ view=article\&id=5741:folha-inf ormativa-mortalidade-materna\&Itemid=820>. Acesso em: 28 abr. 2020.

TURATO, Egberto Ribeiro. Métodos qualitativos e quantitativos na área da saúde: definições, diferenças e seus objetos de pesquisa. Rev. Saúde Pública, São Paulo, v. 39, n. 3, p. 507-514, June 2005 . Available from 〈http://www.scielo.br/scielo.php?script=sci_arttext\&pid=So034-89102005000300025\&lng=en\& 
nrm=iso>. access on 15 Dec. 2020. http://dx.doi.org/10.1590/So034-89102005000300025.

VALENZUELA, Carlos y. Anticoncepción de emergencia, Levonorgestrel y embarazo ectópico. Revista Médica Chilena, Chile, v. , n. 133, p.612-613, dez. 2005.

ZUCCHI, Renato Monteiro et al. Gravidez ectópica após uso de contracepção de emergência: relato de caso. Revista Brasileira de Ginecologia e Obstetrícia, [s.1.], v. 26, n. 9, p.741-743, out. 2004. FapUNIFESP (SciELO). http://dx.doi.org/10.1590/soioo-72032004000900oir. 